\title{
Diseño curricular basado en la opinión de expertos: propuesta metodológica para los estudios de grado
}

\author{
José Tovar Jiménez \\ Profesor de la Universidad a Distancia de Madrid, UDIMA (España) \\ josetovar@cef.es / https://orcid.org/0000-0001-8760-4218
}

\section{Extracto}

El objetivo principal de este trabajo es contribuir a la mejora de la calidad de la formación universitaria en los estudios de grado a partir de la opinión de expertos en la materia para el diseño curricular de las asignaturas.

Para ello es necesario contar con la opinión de académicos y de profesionales (hombres y mujeres) en activo que hagan posible una formación en conocimientos y habilidades acorde a las exigencias de un mercado de trabajo en continuo cambio.

Asimismo, es necesario alcanzar un consenso entre los profesionales implicados que permita identificar los aspectos esenciales. Para este fin, se realizaron entrevistas en profundidad a un grupo reducido de expertos y, a continuación, se recabó la opinión de un panel más amplio de participantes a través del método Delphi.

El resultado final es un diseño curricular para la enseñanza de Derivados Financieros en los estudios del grado de Administración y Dirección de Empresas (ADE), en cuanto a contenidos, competencias y sistema de enseñanza y evaluación que se consideran más eficaces.

Palabras clave: diseño curricular; competencias profesionales; proceso de enseñanza-aprendizaje; entrevistas en profundidad; método Delphi. 


\title{
Curriculum design based on expert opinion: methodological proposal for undergraduate studies
}

\author{
José Tovar Jiménez
}

\section{Abstract}

The main objective of this work is to contribute to the improvement of the the quality of university education in undergraduate studies based on the opinion of experts in the field for the curricular design of the subjects.

For this, it is necessary to have the opinion of academics and professionals (men and women) in active service who make possible a training in knowledge and skills in according to the demands of a constantly changing job market.

Likewise, it is necessary to reach a consensus among the professionals involved, which allows the essential aspects to be identified. To this end, in-depth interviews were conducted with a small group of experts and then the opinion of a larger panel of participants was obtained using the Delphi method.

The final result is a curricular design for the teaching of Financial Derivatives in the Degree of Business Administration and Management, in terms of content, competencies and the teaching and evaluation system that are considered more effective.

Keywords: curriculum design; professional skills; teaching-learning process; in-depth interviews; Delphi method. 


\section{Sumario}

1. Introducción

2. Metodología

2.1. Colectivos de profesionales participantes en la consulta

2.2. Metodología de la investigación

2.2.1. Entrevistas en profundidad

2.2.2. El método Delphi de consulta a hombres y mujeres expertos

2.2.2.1. Características del método Delphi

2.2.2.2. Desarrollo del método Delphi

3. Resultados

3.1. Aspectos generales del estudio de los derivados financieros en grado

3.1.1. Profesionales participantes en la elaboración del plan de estudios

3.1.2. Inclusión de los derivados en los estudios de grado

3.1.3. Contenidos que se deben incluir en el plan de estudios

3.2. Análisis de las competencias en la materia Derivados Financieros

3.3. Aspectos relacionados con el proceso de enseñanza-aprendizaje

3.3.1. Adecuación de los métodos de enseñanza

3.3.2. Sistema de evaluación

4. Discusión y conclusiones

Referencias bibliográficas 


\section{Introducción}

En la época actual, en la que los conocimientos cambian de forma acelerada y se difunden con la misma rapidez, los sistemas educativos deben ser objeto de una profunda revisión, y solo tiene sentido pensar en una formación curricular en términos de competencias y capacidades que los estudiantes deben desarrollar a lo largo de toda la vida.

Los continuos cambios tecnológicos suponen profundas transformaciones en la concepción de los puestos de trabajo y en las exigencias para poder desempeñar las tareas. Así, la empleabilidad se asocia de forma obligada a las

Los planes de estudio deben ser flexibles y estar relacionados con los intereses sociales y económicos competencias que toda persona debe alcanzar de forma continua, con el fin de poder desarrollarse en un ámbito tan dinámico y competitivo como el actual. Por este motivo, los planes de estudio deben ser flexibles y estar relacionados con los intereses sociales y económicos; con contenidos vinculados con la producción y que permitan la transferencia de conocimientos de las instituciones educativas a las empresas.

Para este autor las competencias se deben relacionar con las tareas laborales y han de tener como referencia a aquellos empleados (hombres y mujeres) que son particularmente exitosos. Y la forma de identificar estas competencias es conocer las necesidades del mercado a través de las empresas, en función de los perfiles que manejan y formando a profesionales con conocimientos actualizados, acordes a la realidad empresarial.

En esta línea, Yániz (2008) considera que se debe adoptar un enfoque profesionalizador, que tome como punto de referencia el perfil profesional centrado en el aprendizaje y no en el contenido. De esta forma, el criterio principal a la hora de diseñar el plan de estudios será el conjunto de competencias que se pretende adquirir, lo que condicionará la metodología de aprendizaje y la selección de contenidos más adecuados. Por este motivo, se precisa de un plan de actuación de forma que el diseño curricular permita un auténtico proyecto formativo integrado, diseñado en su totalidad, que tenga como finalidad «obtener mejoras en la formación de las personas que participan en él». De este modo, la transformación de los planes de estudio en proyectos de formación exige una planificación compleja que se inicia con la definición de los objetivos y los resultados esperados; con una selección de metodologías acordes a esos objetivos, que permita alcanzarlos de manera razonable; con una selección de contenidos; y, por último, con un plan de evaluación de los procesos y resultados. 
Sin embargo, el trabajo de Torres-Coronas y Vidal-Blasco (2015) pone de manifiesto las diferencias significativas entre el nivel competencial percibido por los empleadores y el de los estudiantes, en lo que se refiere a competencias digitales. Apuntan, entre las posibles causas del desajuste, dos factores:

- El hecho de que existe cierto desconocimiento por parte del alumnado de las competencias profesionales que el mercado demanda.

- La mayoría de los empleadores no buscan un perfil específico, sino una persona graduada con un conjunto específico de competencias.

Unos años antes, Freire-Seoane et al. (2013) realizaron una investigación para profundizar en el conocimiento de las competencias profesionales de los egresados de la Universidad de A Coruña en relación a las demandadas por los empresarios de la provincia. En ella se evidencia la discrepancia en la valoración realizada por los egresados y por los empresarios, siendo las competencias personales y las instrumentales en las que existe mayor brecha. Estos resultados conducen a los autores a proponer la realización de estudios sistemáticos con la finalidad de que el sistema universitario tenga información actualizada sobre la realidad cambiante del mercado laboral. Hynes y Kwon (2018), en un estudio con ayuda de la técnica Delphi, ponen de manifiesto la importancia de la competencia de presentación oral del alumnado. Asimismo, Herrero (2018) puso de manifiesto la brecha existente entre las habilidades profesionales que se les exigen a los periodistas en el mercado laboral actual y las que realmente se ensañan en los estudios de grado.

En este sentido, Quintana et al. (2016) consideran que para promover la empleabilidad de los graduados hay que identificar el tipo de competencias que van a ser demandadas en el mercado laboral, ahora y en el futuro. También se muestran partidarios de que los empleadores (hombres y mujeres), las organizaciones empresariales y grupos específicos de profesionales participen en el diseño de los planes de estudio, así como en la definición de contenidos de las actividades de enseñanza e, incluso, en el material didáctico que se ha de utilizar. Proponen incluso un aprendizaje mixto, con la participación de estos grupos en el proceso de enseñanza.

El trabajo de Fullana et al. (2011) señala que los planes de estudios deben reestructurarse a partir de la identificación de las competencias profesionales que los estudiantes deben adquirir, de las aportaciones de los profesionales en ejercicio y de las instituciones que ofrecen formación. Así pues, resulta fundamental conocer la opinión

Con la finalidad de promover la empleabilidad de los graduados hay que identificar el tipo de competencias que van a ser demandadas en el mercado laboral
Resulta fundamental conocer la opinión de los profesionales acerca de los requisitos que ha de tener la formación que debe recibir el alumnado universitario 
de los profesionales acerca de los requisitos que ha de tener la formación que debe recibir el alumnado universitario, de forma que le facilite su integración en el mercado laboral. Para un correcto diseño o rediseño de los planes de estudio es necesario contar con el punto de vista de todas las partes implicadas en el proceso: estudiantes, docentes, empleadores y profesionales. Y el trabajo realizado por Pozo y Bretones (2015), a través de entrevistas entre expertos en calidad educativa, concluye que para implementar las actuaciones que permitan la mejora de los nuevos títulos universitarios se debe contar con profesionales externos.

Así pues, y de acuerdo con los postulados de Fullana et al. (2011), dos son los aspectos que se han de tener en cuenta principalmente a la hora de estudiar esta cuestión:

- El conocimiento de las necesidades formativas de cualquier profesional se debe realizar teniendo en cuenta la opinión de los formadores y la experiencia de los profesionales en ejercicio.

- La necesidad de obtener un consenso entre profesionales es fundamental para identificar los aspectos esenciales de una formación de calidad.

Con objeto de resolver las cuestiones previamente señaladas, se plantea el siguiente objetivo principal de investigación: analizar la mejora de la calidad de la formación universitaria en la asignatura de derivados financieros. Como objetivos específicos de este estudio, se proponen:

- Valorar la conveniencia/necesidad de incluir esta materia en el plan de estudios del grado universitario.

- Identificar los conocimientos, las competencias, las habilidades y las aptitudes personales que se consideran prioritarias para ejercer sus funciones y que estén ajustadas a las demandas del mercado.

- Revisar los sistemas metodológicos que hay que seguir en el proceso de enseñanza-aprendizaje, con especial atención a dos aspectos:

- Métodos de enseñanza que se consideran apropiados.

- Sistemas de evaluación que se van a emplear.

- Realizar una propuesta del contenido curricular de la asignatura Derivados Financieros en el grado en ADE. 


\section{Metodología}

El diseño de la investigación se plantea desde una perspectiva holística, procurando una visión del tema en su globalidad, incluyendo a los diferentes colectivos implicados en esta tarea. Se pretende reflejar la percepción de todos ellos sobre las necesidades formativas que el profesional necesita para el desempeño de su actividad laboral ordinaria. Este apartado será el punto de partida para una planificación eficiente de las actuaciones formativas orientadas a mejorar la formación de profesionales.

\subsection{Colectivos de profesionales participantes en la consulta}

La heterogeneidad del panel puede dar mejores resultados que la homogeneidad, particularmente cuando existe una pluralidad de participantes de diferente naturaleza. Además, esta diversidad mejora la credibilidad y la aceptación de los resultados de la investigación.

Para la selección de las personas participantes se ha procurado garantizar la mayor diversidad posible en cuanto a universidades, empresas y representantes de la Administración pública. La intención principal de este estudio de investigación es recoger la opinión de responsables con diferentes perfiles y áreas de conocimiento.

La intención principal de este estudio de investigación es recoger la opinión de responsables con diferentes perfiles y áreas de conocimiento

De acuerdo con los criterios de selección antes mencionados, de cara a esta investigación se va a prestar atención a aquellos grupos que, por su relación directa con los derivados financieros, pueden aportar información que permita alcanzar los objetivos propuestos en este estudio. En este sentido, las entrevistas se realizarán a:

- Docentes de las diferentes universidades madrileñas que imparten este material.

- Supervisores, encargados de establecer el marco normativo y velar por su cumplimiento.

- Profesionales de banca que operan con ellos en su actividad ordinaria.

\subsection{Metodología de la investigación}

El desarrollo del estudio ha seguido un proceso de análisis estructurado en dos fases:

- Realización de entrevistas en profundidad.

- Aplicación del método Delphi a un grupo de expertos. 
Partiendo de un esquema predefinido, se opta por comenzar con entrevistas no estructuradas con preguntas abiertas y simples en torno a la idea principal que es objeto de la investigación, que permiten una mejor adaptación a las necesidades de la investigación y a las características de las personas entrevistadas.

Se ha elegido esta metodología con el fin de obtener una visión general sobre la materia objeto de estudio, lo que permitirá definir el cuestionario que será empleado en la consulta que se realizará más adelante al panel de expertos.

A continuación, y con la intención de recabar el conocimiento y la experiencia que los profesionales que trabajan con estos productos pueden aportar, se aplica la técnica Delphi, que busca el consenso de personas expertas sobre las cuestiones planteadas para identificar lo que actualmente demanda el sector financiero y, en última instancia, realizar una propuesta de plan de estudios sobre la materia Derivados Financieros en los estudios de grado en ADE.

En los siguientes subepígrafes se desarrollan los aspectos de cada una de las técnicas empleadas en este estudio de investigación.
En esta investigación se aplica la técnica Delphi, que busca el consenso de personas expertas sobre las cuestiones planteadas para identificar lo que actualmente demanda el sector financiero y, en última instancia, realizar una propuesta de plan de estudios

\subsubsection{Entrevistas en profundidad}

Se comienza con entrevistas no estructuradas en torno a la idea principal objeto de la investigación. No se trata de un cuestionario cerrado, como el esquema de la encuesta habitual, sino que, por el contrario, se plantea como un cuestionario abierto.

El guion de las entrevistas se concreta en seis bloques con los que se pretende conseguir una visión general de las cuestiones que finalmente se incluyen en el cuestionario que se va a plantear al panel de expertos en el método Delphi posterior:

- Visión general de la formación universitaria en los grados en Economía y Empresa.

- Características generales de los derivados financieros.

- Competencias profesionales.

- Diseño curricular.

- Metodología de enseñanza.

- Sistemas de evaluación. 
Se han llevado a cabo 11 entrevistas personales: 5 a profesores de diferentes universidades, 3 a profesionales financieros de la banca y 3 a reguladores financieros. En ningún momento se ha pretendido una representación estadística de los perfiles seleccionados.

Las personas participantes en esta ronda de entrevistas no deben formar parte del panel de expertos del método Delphi.

\subsubsection{El método Delphi de consulta a hombres y mujeres expertos}

Para López-Gómez (2018), la vigencia social y metodológica de la técnica Delphi en diversas áreas de investigación social queda avalada por la gran cantidad de investigaciones que la han utilizado, muchas de ellas en el ámbito de la educación.

En esta misma línea, Cabero e Infante (2014) destacan en su trabajo cómo el uso del método Delphi se extiende en diversas áreas científicas y, en particular, en comunicación y educación. En concreto, en el ámbito educativo se ha aplicado a diversas temáticas: validación de cuestionarios, evaluación de necesidades, evaluación de la calidad didáctica, e-learning y en el campo de las tecnologías de la información y comunicación (TIC).

Son variadas las aplicaciones de esta metodología en el ámbito de la enseñanza. Así, por ejemplo, López et al. (2018) también recurren a la metodología Delphi para la validación de un cuestionario de valoración de observatorios digitales con dos rondas realizado entre docentes y personas expertas en TIC. También Bailie (2015) utiliza esta técnica para validar el consenso entre docentes y alumnado sobre cuestiones referidas a la educación superior online. En un estudio reciente, Victoria et al. (2020) utilizan esta técnica para el empleo del aprendizaje colaborativo en la enseñanza superior y Torres-Gordillo y Vidal-Blasco (2020) muestran los resultados de su investigación en cinco universidades españolas, centrada en la percepción del personal docente universitario sobre la implementación de la metodología ECO (explorar, crear y ofrecer) para conocer el impacto que esta tiene tanto en la enseñanza como en el aprendizaje. Por su parte, Lnenicka et al. (2020) emplean el método Delphi para analizar el uso del big data con la finalidad de detectar las habilidades necesarias en el proceso de la enseñanza superior.

Este método también ha sido utilizado para determinar competencias profesionales en diferentes entornos. En el trabajo de Fullana et al. (2011) se aplica en el área de competencias docentes del profesorado. También se puede encontrar la aplicación de esta metodología en la realización de un estudio para obtener las claves para desarrollar la competencia científica de los nuevos docentes de primaria en el ámbito de la enseñanza de las ciencias, a partir de las percepciones de una muestra de docentes en activo, y, asimismo, se recurre a la metodología participativa, de consulta y consenso con expertos como un mecanismo que permite valorar el ajuste de las competencias genéricas de las personas egresadas en relación con las necesidades demandadas por el entorno empresarial. 
De Juanas et al. (2016) realizan un estudio orientado a obtener las claves para desarroIlar la competencia científica de los nuevos docentes de primaria en el ámbito de la enseñanza de las ciencias a partir de las percepciones de una muestra de docentes en activo. Las autoras Rosales y Cabrera (2017) también hacen uso de esta metodología para evaluar las competencias de las personas egresadas en los estudios de ingeniería y, en 2017, se recurre a esta técnica para explicar la realidad educativa en Canarias, intentando combinar y contrastar los diferentes puntos de vista de los sujetos implicados, a fin de entender de un modo más global la realidad sobre los bajos resultados educativos en las islas. En su trabajo, Chuenjitwongsa et al. (2017) recurren al método Delphi con el objetivo de diseñar un plan de estudios para estudiantes de odontología a partir del aprendizaje basado en competencias. Yağci y Güneyli (2018) han empleado esta técnica para establecer los contenidos de la formación del profesorado en TIC y Homberg et al. (2020), para determinar las competencias profesionales en el ámbito de la salud.

Así pues, la investigación científica y tecnológica relacionada con el método Delphi se encuentra en una etapa de plena actividad y desarrollo. Da cuenta de ello el elevado volumen de resultados publicados en revistas especializadas, con un marcado crecimiento exponencial (Cruz y Rúa, 2018). Esta técnica ha mostrado tener una gran eficacia y potencialidad como método de investigación, y su difusión y desarrollo se han incrementado en las últimas décadas. En especial, se considera que es una herramienta muy útil en el ámbito educativo, ya que permite obtener información acerca de la realidad de cualquier tema de acuerdo con las opiniones de un variado grupo de participantes conectados con el objeto de la investigación.

\subsubsection{Características del método Delphi}

Para Landeta (1999), las principales características de este método son las siguientes:

- Proceso iterativo. A través de al menos dos rondas de consultas sobre una misma cuestión, se pregunta a las personas expertas, dándoles la posibilidad de reconsiderar su opinión inicial en función de la opinión agregada de las respuestas del panel. En este proceso, las contestaciones de las personas participantes tienden a converger, llegando a su fin cuando se obtiene el consenso o las opiniones se estabilizan.

- Anonimato de las personas participantes. Ninguna de las personas participantes conoce la identidad del resto de miembros del panel, ni tampoco las respuestas particulares de cada uno de ellos. Las ventajas que esto conlleva, según Cabero e Infante (2014), son:

- Evita la influencia de determinados líderes.

- Posibilita el cambio de opinión de los participantes.

- Impide la interacción del grupo. 
- Retroalimentación controlada. El intercambio de información entre las personas participantes se realiza a través del coordinador del estudio, asegurando la correcta interpretación de las preguntas formuladas y eliminando la información que no sea importante. Al inicio de cada ronda se da a conocer la posición general del grupo sobre la cuestión analizada, así como las aportaciones y sugerencias de los expertos y cualquier otra información adicional que el coordinador considere oportuno. Esta interacción justifica la superioridad de las técnicas grupales frente a las individuales, en la medida que permite llegar a un juicio global del conjunto de participantes, evitando la confrontación directa.

- Respuesta estadística del grupo. El objetivo es conseguir un tratamiento estadístico de las respuestas a través de medidas de posición central ${ }^{1}$ y de dispersión ${ }^{2}$. Se trata de que los individuos participantes conozcan tanto el punto de vista de la mayoría como la totalidad de las opiniones del grupo, mostrando el grado de acuerdo que se ha obtenido. Con la respuesta estadística de grupo se consigue:

- Garantizar que las opiniones de todos los miembros estén presentes en la respuesta grupal.

- Reducir la presión hacia la conformidad.

\subsubsection{Desarrollo del método Delphi}

La metodología propuesta para esta investigación toma como referencia, entre otros, los trabajos de Gutiérrez et al. (2003) y Fullana et al. (2011) y se debe estructurar en los siguientes apartados:

- Marco de trabajo.

- Muestra de expertos.

- Proceso iterativo de los cuestionarios.

- Interpretación de los resultados.

El estudio se lleva a cabo a través del diseño, la validación y la ejecución de un proceso de consulta iterativa estructurado en dos rondas. En la figura 1 se muestra la secuencia que hay que seguir para la implementación del proceso, donde se destaca el papel del moderador y la retroalimentación que permite el consenso de opinión de las personas expertas consultadas (Gutiérrez et al., 2003):

\footnotetext{
1 Se emplea la media aritmética.

2 Se emplea la desviación típica.
} 
Figura 1. Esquema general del proceso Delphi

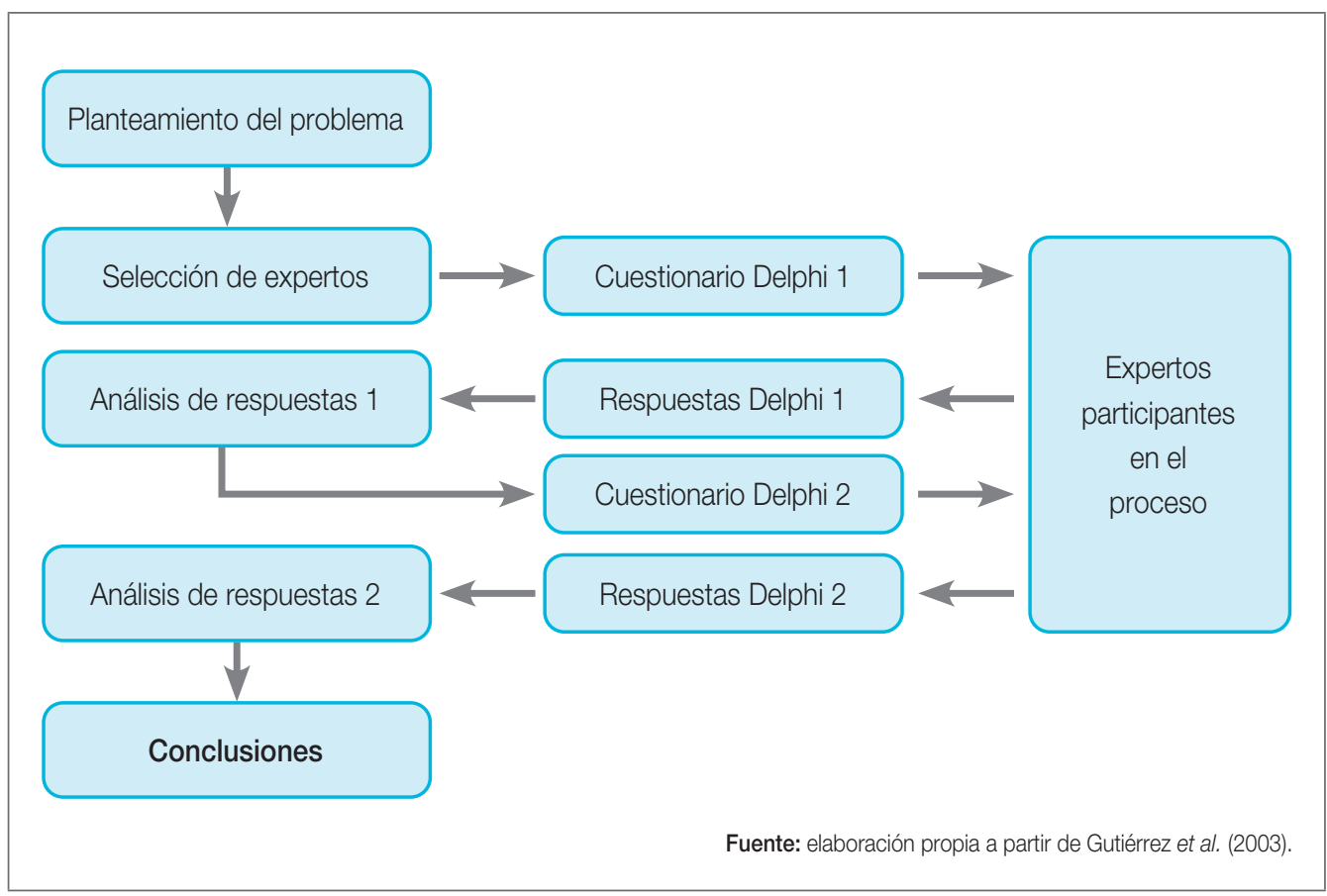

A continuación, se desarrollan cada uno de los pasos a seguir:

\section{A) Selección de hombres y mujeres expertos}

Se trata de un aspecto crucial en la investigación, habida cuenta de que la calidad del proceso y de los resultados obtenidos dependen de una adecuada selección de los sujetos intervinientes (López-Gómez, 2018). Los criterios para la selección de las personas expertas participantes, de acuerdo con este autor, han sido:

- Inclusión. La naturaleza de la técnica elegida hace imposible la selección aleatoria.

- Experiencia en relación con los objetivos de la investigación. No se busca nominar a personas expertas que tengan un número determinado de publicaciones sobre el tema de la investigación. Por el contrario, se buscan individuos que sean especialistas en su campo, a los que se les requiere conocimiento basado en la práctica y experiencia actualizada.

- Multisectorialidad. Se trata de incluir en el panel a personas relacionadas con el tema de investigación desde diferentes puntos de vista, lo que permitirá el contraste de opiniones y la validación de los resultados finales. 
- Disposición. Es necesario contactar con individuios expertos que muestren interés por el tema y que estén motivados e ilusionados con la investigación. De esta forma, se garantiza su participación significativa y sostenida en el tiempo.

El primer contacto con estos participantes se realizó por vía telefónica o correo electrónico y, una vez explicado de forma muy breve las líneas generales de la investigación, se les solicitó su colaboración. Posteriormente, a través de e-mail, se les informó más detalladamente sobre los objetivos del estudio y los pormenores de la metodología que se iba a seguir (ejecución a través de un cuestionario online, así como el tiempo aproximado de respuesta). También se les resolvieron las dudas que en algunos casos se plantearon.

Una vez concluida la investigación y analizados los resultados obtenidos, a todos los participantes se les remitieron por correo electrónico las conclusiones finales y se les agradeció su participación.

\section{B) Número de hombres y mujeres expertos}

En esta técnica no hay normas sobre el número de participantes que deben incluirse para garantizar la validez estadística de la muestra. Para Landeta (1999), en general, se considera que no pueden ser menos de 7 y el máximo lo sitúa en torno a los 30 . El estudio de las referencias bibliográficas consultadas revela que la mayoría de los estudios Delphi han empleado entre 15 y 20 expertos.

En esta investigación el número previsto de participantes fue de 58, que se considera más que suficiente de acuerdo con la bibliografía consultada, destacando la homogeneidad de las muestras de expertos, en las que cabe destacar una mayor participación del grupo de profesionales de banca y reguladores y supervisores bancarios (véase cuadro 1).

Cuadro 1. Distribución de expertos participantes al inicio y al final del estudio

\begin{tabular}{lcc}
\multicolumn{1}{c|}{ Grupo de expertos } & Inicio del estudio & Final del estudio \\
\hline Docentes & $13(22,42 \%)$ & $13(23,21 \%)$ \\
\hline Profesionales de banca & $24(41,38 \%)$ & $23(41,07 \%)$ \\
Reguladores y supervisores & $21(36,21 \%)$ & $20(35,72 \%)$ \\
\hline Total & 58 & 56
\end{tabular}


La distribución inicial por sexos indica una mayor participación de hombres $(72,41 \%)$ frente al $27,59 \%$ de mujeres. Dentro del colectivo femenino hay que destacar que tan solo 3 de las 16 mujeres que han sido consultadas (5,17\% del total de participantes) ocupan puestos académicos, estando más equilibrada su presencia en los otros dos grupos (6 y 7 , de profesionales de banca y reguladores, respectivamente).

\section{C) Calidad del panel}

Para López-Gómez (2018) la calidad del panel está justificada por las publicaciones realizadas o por la experiencia profesional de sus integrantes. Sin embargo, en esta selección puede tenerse en cuenta el puesto de trabajo que ocupan y la trayectoria profesional, sin necesidad de someter a los individuios participantes a ningún índice de competencia experta como proponen diversos autores (Cabero e Infante, 2014).

Como medida para garantizar esta experiencia, en el primer cuestionario se incluyen preguntas para que cada experto indique: perfil profesional, años de desempeño de su actividad, experiencia docente (para el caso de profesionales) y, por último, el grado de conocimiento sobre la materia. Este procedimiento de autovaloración permite descartar, si se estima conveniente, la participación de aquellos que no reúnan los niveles mínimos de calidad requeridos. De esta forma, se sigue la línea más usual en la práctica del método Delphi (López-Gómez, 2018).

La experiencia de los participantes queda sobradamente acreditada a tenor de los años de desempeño en sus tareas, que se aprecia en el cuadro 2. Destaca el sector académico, cuyos años de profesión casi triplican a la de reguladores y supervisores.

Cuadro 2. Experiencia de las personas participantes

Grupo de expertos

Docentes

Profesionales de banca

Reguladores y supervisores

Total
Media (años)

23,83

16,58

8,90

Fuente: elaboración propia.

Solo 6 participantes tienen una experiencia inferior a los 2 años y los que acumulan más de 10 años de actividad (véase figura 2) son mayoría (37). No obstante, en este caso, la falta de experiencia puede ser compensada por su cercanía al periodo de formación 
universitaria, lo que resulta útil para poder tener un conocimiento más profundo de las modificaciones que ha supuesto el cambio metodológico que se ha producido con la puesta en funcionamiento del Espacio Europeo de Enseñanza Superior (EEES).

Figura 2. Años de experiencia del panel de expertos

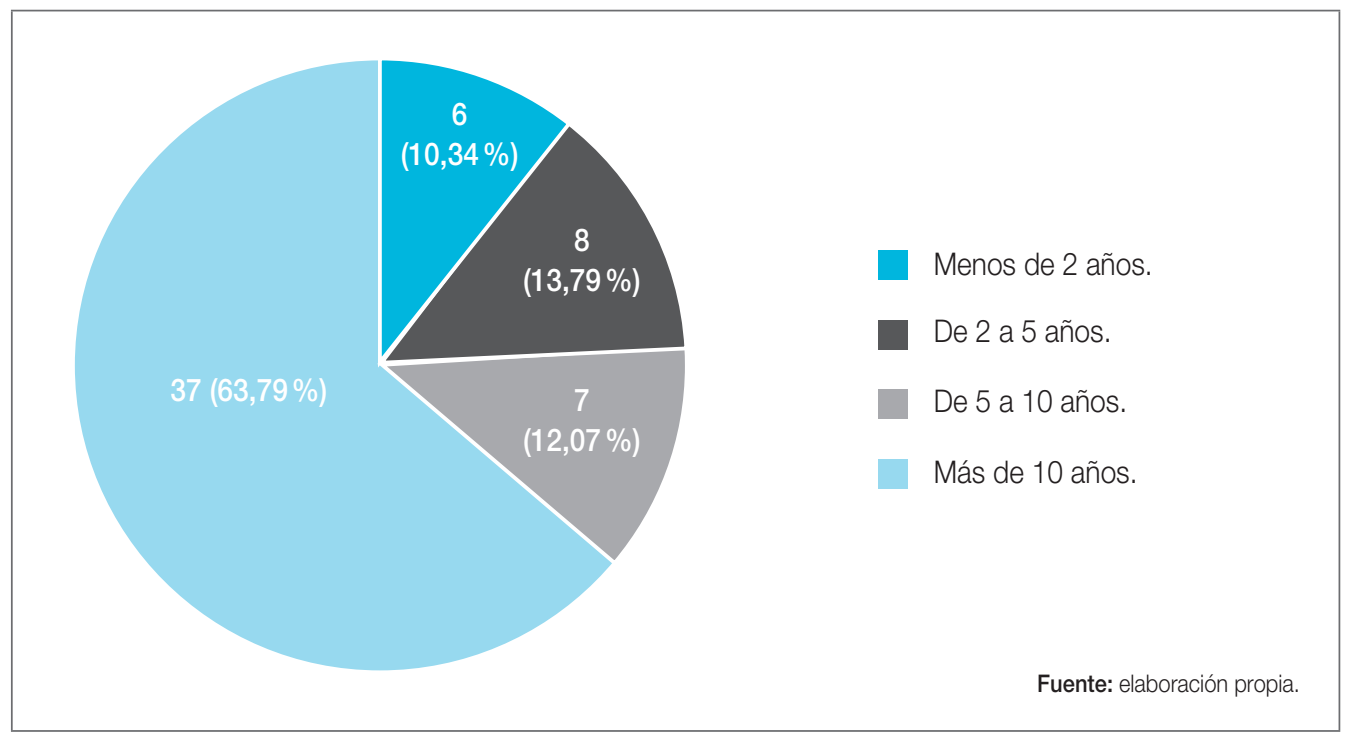

También hay que destacar la elevada experiencia docente de los expertos no académicos. En el panel figuran 17 profesionales de banca y 13 reguladores que confirman su experiencia como docentes universitarios en temas relacionados con los derivados financieros. De esta manera, junto a los 13 docentes universitarios, el número de expertos con experiencia en la enseñanza de la materia asciende a 43 (74,14\% del panel).

\section{D) Desarrollo del cuestionario}

Se ha procedido a elaborar un único cuestionario para todos los docentes y profesionales seleccionados. La realización de una única encuesta para los diferentes grupos de expertos pretende poner de manifiesto las posibles diferencias de opinión entre ellos, realizando los oportunos análisis de contenidos que permitan conocer las coincidencias y discrepancias en las cuestiones analizadas.

Se ha considerado conveniente realizar una prueba piloto con un grupo de participantes (que no forman parte del panel de expertos) para conocer su opinión sobre las siguientes preguntas: ¿ha tenido dificultad para entender las preguntas?, ¿considera oportuno incluir alguna pregunta adicional?, ¿considera que debe eliminarse alguna pregunta ?, ¿le ha parecido 
demasiado extenso? y ¿qué mejoras introduciría? Obtenidas las respuestas, se procede a incorporar las aportaciones realizadas para que el cuestionario quede definitivamente validado.

El siguiente paso ha consistido en preparar el cuestionario a través de la plataforma online LimeSurvey, estableciendo un plazo aproximado de dos semanas para la obtención de las respuestas y con un intervalo entre cada ronda suficiente para el tratamiento de la información.

El formulario ha quedado estructurado en tres apartados con una breve introducción, en la que se describe la finalidad de la investigación que se va a llevar a cabo y algunas pautas para su realización. Los bloques del cuestionario han sido:

- Aspectos generales de los derivados financieros.

- Proceso de enseñanza-aprendizaje.

- Perfil profesional del encuestado.

El cuestionario ha quedado configurado en torno a 4 dimensiones y 34 preguntas, con un total de 84 ítems. Estas dimensiones son:

- Organización de la asignatura.

- Competencias.

- Métodos de enseñanza.

- Estrategias evaluativas.

La mayoría de las preguntas han sido de tipo escalar, con valores comprendidos entre 1 y 4 . El cuestionario también incluye preguntas abiertas en las que se invita a los individuos participantes a compartir los comentarios que estimen oportunos. En el cuadro 3, se muestra la distribución.

\section{Cuadro 3. Distribución de las preguntas del primer cuestionario}

Tipo de pregunta

Identificación del experto

Preguntas cerradas (con escala de valoración)

Preguntas abiertas

Total

\section{Número de variables}

6

19

9 


\section{E) Proceso iterativo en dos rondas}

La realización de las entrevistas en profundidad, efectuadas en la fase anterior de esta investigación, permite la construcción del cuestionario que será objeto de análisis en el Delphi y posibilita la finalización del procedimiento en dos etapas, a diferencia de otros estudios realizados con esta metodología que se inician con una primera ronda de preguntas abiertas.

Las opiniones de las personas encuestadas son volcadas a una hoja de cálculo para su tratamiento estadístico. Se calculan las frecuencias relativas de cada una de las categorías, las medidas de tendencia central y de dispersión para cada uno de los ítems y se ordenan de acuerdo con estos indicadores.

En la segunda ronda se envía de nuevo el cuestionario, del que se ha eliminado el bloque de preguntas referidas al perfil profesional y en el que, en algunas preguntas, se han incorporado algunos ítems más, de acuerdo con las recomendaciones realizadas por los expertos en la ronda anterior. En esta segunda vuelta se les comunica a las personas participantes la frecuencia de las respuestas y los valores medios del grupo para cada uno de los ítems de la ronda inicial. De nuevo, se les pide que respondan a las mismas preguntas del cuestionario anterior, solicitando que confirmen su primera respuesta o, por el contrario, la rectifiquen de acuerdo con las opiniones de los demás panelistas. También se les da la opción de que argumenten su decisión de mantener o modificar su respuesta.

En las dos rondas se fija una fecha máxima de 15 días para la cumplimentación del cuestionario, aunque, con la intención de asegurar la máxima participación, no se tiene que ser demasiado estricto sobre este particular. Para recordarles y confirmar su paticipación en el estudio, se contacta con los participantes por vía telefónica o a través de correos electrónicos.

\section{F) Escala de medición}

De acuerdo con la práctica generalizada del uso de las escalas tipo Likert en los campos de la psicología y educación (Bisquerra y Pérez-Escoda, 2015), en el cuestionario se empleó una escala Likert para mostrar el grado de acuerdo o desacuerdo con la cuestión planteada.

Se toma como referencia el estudio sobre la incorporación de las TIC en la práctica docente de los autores George y Trujillo (2018) a la hora de diseñar y validar el cuestionario de la investigación Delphi y se decide usar la escala Likert con valores de 1 a 4 puntos para cada ítem del cuestionario.

Para ello se solicitará a los expertos que valoren de acuerdo con una escala Likert de 1 a 4 en cuatro bloques:

- Aspectos generales relacionados con la asignatura ( 1 = Nada de acuerdo; 2 = En desacuerdo; 3 = De acuerdo; 4 = Totalmente de acuerdo). 
- Competencias transversales y específicas que el alumnado deba adquirir ( 1 = Nada importante; 2 = Poco importante; 3 = Importante; 4 = Muy importante).

- Aspectos de la asignatura, tales como el carácter obligatorio u optativo, la importancia relativa que deben tener los diferentes métodos de enseñanza, los sistemas de evaluación propuestos, etc. Al respecto, se emplea una escala Likert de 1 a 4 (1 = Nada eficaz; 2 = Poco eficaz; 3 = Eficaz; 4 = Muy eficaz).

- Cuestiones referidas al perfil profesional del experto, con preguntas referidas a «tipo de organización en la que desempeña su actividad profesional», «características de su actividad profesional», «años de experiencia en el puesto» y «su posible experiencia docente para el caso de profesionales».

\section{G) Finalización del método Delphi}

En la práctica habitual que pone de manifiesto los resultados de la revisión bibliográfica llevada a cabo por Steurer (2011), se fija de antemano la finalización del proceso en dos rondas con independencia de los resultados obtenidos. La razón de esta decisión estriba en lo complicado que resulta contar con la colaboración de los expertos en una tercera ronda.

Landeta (1999) considera el "consenso»33 entre las opiniones de los panelistas como el criterio clásico utilizado desde los orígenes de este método para determinar la finalización del proceso. No obstante, aclara que esta forma de concluir la consulta supone el riesgo de alcanzar acuerdos ficticios forzados por la propia metodología de la técnica. Por este motivo establece la "estabilidad de las respuestas» 4 como criterio recomendable para determinar la finalización, correspondiendo al individuo investigador decidir el nivel de exigencia necesario para que la convergencia sea aceptable. Para medir el nivel de estabilidad de los resultados este autor propone dos soluciones: la «estabilidad grupal» de la respuesta del conjunto de los expertos y la «estabilidad individual», analizando la opinión particular de cada experto a lo largo del proceso.

De acuerdo con García y Palomares (2012), y dado que la elección de los expertos no es de forma aleatoria, sino que responde al grado de especialización del encuestado, cuando un ítem, en una pregunta de respuesta múltiple, alcanza al menos el $50 \%$ de las respuestas o cuando una pregunta acumula el $70 \%$ de las respuestas, se entiende que hay consenso.

3 El «consenso» es el grado de convergencia de las estimaciones individuales, que se alcanza cuando las opiniones presentan un grado aceptable de proximidad (rango intercuartílico reducido) (Landeta, 1999).

4 La «estabilidad de respuestas» implica la no variación significativa de las opiniones de los expertos en rondas sucesivas, independientemente del grado de convergencia alcanzado (Landeta, 1999). 
Lo que proponemos es analizar las diferencias entre los resultados obtenidos en ambas rondas. Para medir el nivel de estabilidad grupal, que considera la respuesta conjunta del grupo de expertos, se empleará la variación del rango intercuartílico relativo (variación RIR) propuesto por Landeta (1999). El «RIR» se define como la diferencia entre el tercer y primer cuartil, dividido por la mediana $(Q 3-Q 1) / Q 2$. La razón de esta elección es doble: por una parte, con la intención de establecer un límite de aceptabilidad único para todas las preguntas y, por otra, porque no tiene en cuenta los valores extremos, por lo que se evita la influencia de los mismos.

La disminución del RIR sugiere que el método Delphi propicie un proceso de reflexión que lleva a un número significativo de expertos a modificar su opinión inicial (Landeta, 1999).

\section{Resultados}

A continuación, se indican los resultados obtenidos en la investigación. Estos se han agrupado por bloques temáticos, respondiendo a los objetivos del estudio.

\subsection{Aspectos generales del estudio de los derivados financieros en grado}

Dentro de este apartado se muestran las opiniones del panel de expertos en relación a los siguientes aspectos:

- Profesionales que deben participar en la elaboración del plan de estudios de este material.

- Inclusión de la materia en los planes de estudios, así como su carácter optativo u obligatorio.

- Contenidos de la asignatura y número de ECTS (European credit transfer and accumulation system) asignados.

\subsubsection{Profesionales participantes en la elaboración del plan de estudios}

Para poder configurar un plan de estudios en lo referente a la materia Derivados Financieros, los expertos consideran que deben ser tenidas en cuenta las opiniones de hombres y mujeres profesionales de banca, académicos, inspectores de entidades de crédito, profesionales de grandes empresas y técnicos de la Comisión Nacional del Mercado de Valores (CNMV) (véase cuadro 4). 
Cuadro 4. Profesionales participantes en la elaboración del plan de estudios

\begin{tabular}{|c|c|c|c|c|c|c|c|c|}
\hline \multirow[t]{2}{*}{ Profesionales } & \multirow[t]{2}{*}{ Media } & \multirow{2}{*}{$\begin{array}{c}\text { Desviación } \\
\text { típica }\end{array}$} & \multirow[t]{2}{*}{$\mathrm{CV}^{*}$} & $1^{* *}$ & $2^{* *}$ & $3^{* *}$ & $4^{* *}$ & $\begin{array}{c}\text { No } \\
\text { contesta }\end{array}$ \\
\hline & & & & \multicolumn{5}{|c|}{$(\%)$} \\
\hline Académicos & 3,47 & 0,599 & 0,173 & 0 & 5,36 & 41,07 & 51,79 & 1,79 \\
\hline Profesionales de banca & 3,64 & 0,614 & 0,169 & 0 & 7,14 & 21,43 & 69,64 & 1,79 \\
\hline Inspectores del Banco de España & 3,11 & 0,724 & 0,233 & 1,79 & 16,07 & 51,79 & 30,36 & 0 \\
\hline Inspectores de seguros & 2,69 & 0,735 & 0,273 & 1,79 & 41,07 & 41,07 & 14,29 & 1,79 \\
\hline Técnicos CNMV & 3,11 & 0,755 & 0,243 & 1,79 & 17,86 & 46,43 & 32,14 & 1,79 \\
\hline Profesionales de grande empresas & 3,33 & 0,793 & 0,238 & 1,79 & 14,29 & 30,36 & 50 & 3,57 \\
\hline Gestores de fondos & 2,67 & 0,839 & 0,314 & 5,36 & 39,29 & 33,93 & 17,86 & 3,57 \\
\hline Operadores de mercados & 3,15 & 0,848 & 0,269 & 3,57 & 17,86 & 35,71 & 39,29 & 3,57 \\
\hline
\end{tabular}

Fuente: elaboración propia

\subsubsection{Inclusión de los derivados en los estudios de grado}

De forma mayoritaria, los expertos coinciden en incluir derivados financieros en el grado en Contabilidad y Finanzas y en el grado en ADE. Para el grado en Economía se produce una disminución de los que están totalmente de acuerdo, aunque la opinión conjunta es a favor de su estudio en el grado (véase cuadro 5).

Cuadro 5. Inclusión de los derivados financieros en los estudios de grado

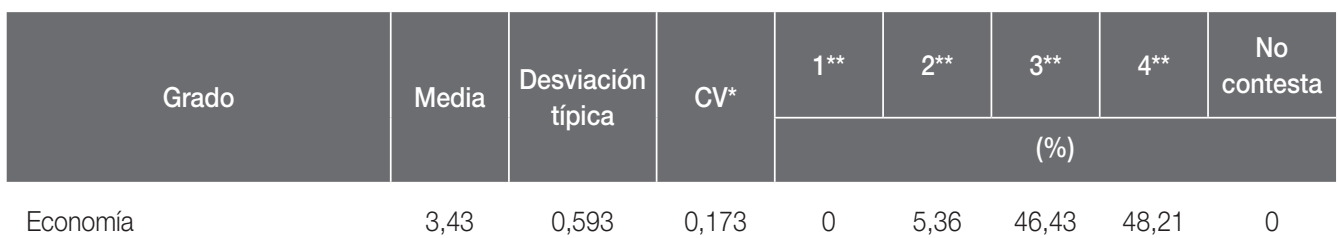




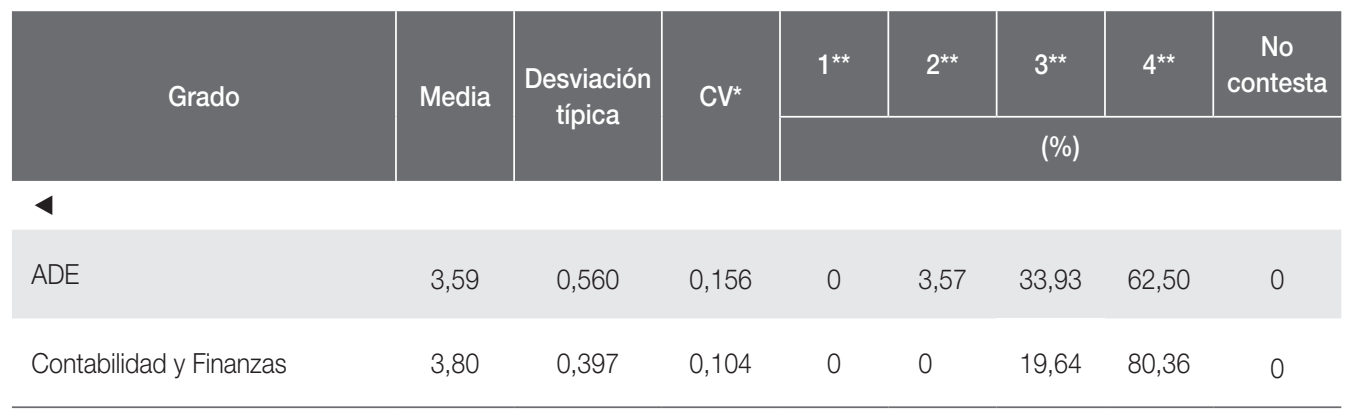

* CV (coeficiente de variación).

** 1 = Nada de acuerdo; 2 = En desacuerdo; 3 = De acuerdo; 4 = Totalmente de acuerdo.

Fuente: elaboración propia.

Las opiniones de los expertos confirman la necesidad de considerar el estudio de los derivados como una asignatura obligatoria en todos los grados. En este sentido, se muestran totalmente de acuerdo para el grado en Finanzas y Contabilidad $(75 \%)$ y en el grado en $\operatorname{ADE}(57,14 \%)$, alcanzado el consenso en el grado en Economía (53,57\% de acuerdo) (véase cuadro 6).

\section{Cuadro 6. Obligatoriedad de la materia Derivados Financieros}

\begin{tabular}{|c|c|c|c|c|c|c|c|c|}
\hline \multirow[t]{2}{*}{ Grado } & \multirow[t]{2}{*}{ Media } & \multirow{2}{*}{$\begin{array}{c}\text { Desviación } \\
\text { típica }\end{array}$} & \multirow[t]{2}{*}{$\mathrm{CV}^{*}$} & $1^{* *}$ & $2^{* *}$ & $3^{* *}$ & $4^{\star *}$ & $\begin{array}{c}\text { No } \\
\text { contesta }\end{array}$ \\
\hline & & & & \multicolumn{5}{|c|}{$(\%)$} \\
\hline Economía & 3,11 & 0,673 & 0,216 & 0 & 17,86 & 53,57 & 28,57 & 0 \\
\hline ADE & 3,54 & 0,566 & 0,160 & 0 & 3,57 & 39,29 & 57,14 & 0 \\
\hline Contabilidad y Finanzas & 3,73 & 0,481 & 0,129 & 0 & 1,79 & 23,21 & 75 & 0 \\
\hline
\end{tabular}

* CV (coeficiente de variación).

** 1 = Nada de acuerdo; 2 = En desacuerdo; 3 = De acuerdo; 4 = Totalmente de acuerdo.

Fuente: elaboración propia.

Por último, en el cuadro 7 se muestran las opiniones de los diferentes grupos de expertos sobre la adecuación de la formación recibida en los estudios de grado. El 58,93\% no están nada de acuerdo con la formación que actualmente reciben, subiendo al $69,23 \%$ entre el profesorado. 
Cuadro 7. Opinión de los expertos (hombres y mujeres) sobre la adecuación de la formación recibida en los estudios de grado

\begin{tabular}{|c|c|c|c|c|}
\hline \multirow{2}{*}{ Opinión de los expertos } & Media & Docentes & $\begin{array}{c}\text { Pofesionales } \\
\text { de banca }\end{array}$ & Reguladores \\
\hline & \multicolumn{4}{|c|}{$(\%)$} \\
\hline Nada de acuerdo & 10,71 & 0 & 18,18 & 9,52 \\
\hline En desacuerdo & 58,93 & 69,23 & 45,45 & 66,67 \\
\hline De acuerdo & 25 & 30,77 & 22,73 & 23,81 \\
\hline Totalmente de acuerdo & 5,36 & 0 & 13,64 & 0 \\
\hline No contesta & 0 & 0 & 0 & 0 \\
\hline
\end{tabular}

Fuente: elaboración propia.

\subsubsection{Contenidos que se deben incluir en el plan de estudios}

De forma clara los expertos confirman la importancia de los futuros y de las opciones, con un notable grado de acuerdo.

Entre los contenidos, solo los productos estructurados alcanzan un grado importante de consenso $(51,79 \%)$. Por el contrario, los warrants y las opciones exóticas quedan rechazadas por las personas encuestadas. En lo que se refiere a los derivados de crédito, una parte importante del panel $(42,86 \%)$ está de acuerdo en incluirlos y una parte importante de los encuestados $(28,57 \%)$ está totalmente en desacuerdo (véase cuadro 8$)$.

Cuadro 8. Contenidos del plan de estudios

\begin{tabular}{|c|c|c|c|c|c|c|c|c|}
\hline \multirow[t]{2}{*}{ Contenidos } & \multirow[t]{2}{*}{ Media } & \multirow[t]{2}{*}{$\begin{array}{c}\text { Desviación } \\
\text { típica }\end{array}$} & \multirow[t]{2}{*}{$\mathrm{CV}^{*}$} & $1^{* *}$ & $2^{* *}$ & $3^{* *}$ & $4^{* *}$ & $\begin{array}{c}\text { No } \\
\text { contesta }\end{array}$ \\
\hline & & & & \multicolumn{5}{|c|}{$(\%)$} \\
\hline Divisas & 3,30 & 0,624 & 0,189 & 1,79 & 3,57 & 57,14 & 37,50 & 0 \\
\hline Futuros & 3,64 & 0,515 & 0,141 & 0 & 1,79 & 32,14 & 66,07 & 0 \\
\hline Opciones & 3,59 & 0,620 & 0,173 & 1,79 & 1,79 & 32,14 & 64,29 & 0 \\
\hline
\end{tabular}




\begin{tabular}{|c|c|c|c|c|c|c|c|c|}
\hline \multirow[t]{2}{*}{ Contenidos } & \multirow[t]{2}{*}{ Media } & \multirow{2}{*}{$\begin{array}{c}\text { Desviación } \\
\text { típica }\end{array}$} & \multirow[t]{2}{*}{$C V^{*}$} & $1^{* *}$ & $2^{* \star}$ & $3^{* *}$ & $4^{* *}$ & $\begin{array}{c}\text { No } \\
\text { contesta }\end{array}$ \\
\hline & & & & \multicolumn{5}{|c|}{$(\%)$} \\
\hline \multicolumn{9}{|l|}{4} \\
\hline $\mathrm{OTC}^{\star \star \star}$ & 3,09 & 0,739 & 0,239 & 1,79 & 17,86 & 50 & 30,36 & 0 \\
\hline Warrants & 2,46 & 0,706 & 0,287 & 7,14 & 44,64 & 42,86 & 5,36 & 0 \\
\hline Opciones exóticas & 2,50 & 0,732 & 0,293 & 5,36 & 48,21 & 37,50 & 8,93 & 0 \\
\hline Derivados de crédito & 3 & 0,756 & 0,252 & 0 & 28,57 & 42,86 & 28,57 & 0 \\
\hline Productos estructurados & 3,05 & 0,692 & 0,227 & 0 & 21,43 & 51,79 & 26,79 & 0 \\
\hline \multicolumn{9}{|c|}{$\begin{array}{l}\text { * CV (coeficiente de variación). } \\
\text { ** } 1 \text { = Nada de acuerdo; } 2 \text { = En desacuerdo; } 3 \text { = De acuerdo; } 4 \text { = Totalmente de acuerdo. } \\
\text { *** OTC (over the counter) }\end{array}$} \\
\hline
\end{tabular}

Fuente: elaboración propia.

A juicio de los expertos, el grado en Contabilidad y Finanzas es el que mayor número de créditos ECTS debe tener, en concordancia con la valoración otorgada a la hora de incluir esta materia en el plan de estudios del grado y en su consideración como asignatura obligatoria. Los expertos consideran que esta asignatura debería contar con 19,71 créditos de media, llegando a un máximo de 40 ECTS y siendo 20 el número más repetido. En el grado en $\mathrm{ADE}$ se reduce el número medio de créditos asignados (16,33 ECTS), manteniéndose el número máximo en 40 ECTS y el mínimo en 20. Y para el Grado en Economía los expertos otorgan un número medio de 13,59 ECTS, aunque la mayoría de los individuos participantes se decantan por asignarle solo 12 (véase cuadro 9).

Cuadro 9. Créditos ECTS para Derivados Financieros en los estudios de grado

\begin{tabular}{|c|c|c|c|c|c|c|c|c|c|c|}
\hline Grado & Media & $\begin{array}{c}\text { Desviación } \\
\text { típica }\end{array}$ & $C V^{*}$ & $\mathrm{Me}^{*}$ & Moda & Mín. & Máx. & Q1 & Q3 & $\begin{array}{c}K= \\
\text { Q3 - Q1 }\end{array}$ \\
\hline Economía & 13,59 & 5,925 & 0,436 & 12 & 12 & 2 & 40 & 11 & 15 & 4 \\
\hline ADE & 16,33 & 6,653 & 0,407 & 18 & 20 & 2 & 40 & 12 & 20 & 8 \\
\hline $\begin{array}{l}\text { Contabilidad y } \\
\text { Finanzas }\end{array}$ & 19,71 & 8,386 & 0,425 & 20 & 20 & 2 & 40 & 13 & 24,5 & 11,5 \\
\hline
\end{tabular}

* CV (coeficiente de variación) y Me (mediana). 
Por colectivos, dentro del panel las respuestas de los docentes son las que arrojan resultados medios más bajos y con menor dispersión. Los expertos coinciden en asignar un mínimo de 2 ECTS en todos los grados, llegando a un máximo de 40 para el grado en Finanzas y Contabilidad.

\subsection{Análisis de las competencias en la materia Derivados Finan- cieros}

El 69,64\% de los expertos está totalmente de acuerdo en la necesidad de diseñar el plan de estudios en torno a la adquisición de competencias profesionales, siendo los profesionales de banca los que están más convencidos.

Al mismo tiempo, en el cuadro 10 se puede observer que tanto los reguladores (60\%) como los profesionales de banca $(52,17 \%)$ coinciden en la necesidad de una redefinición de las competencias de forma considerable, destacando que un $34,78 \%$ de los profesionales de banca son partidarios de una revisión en profundidad. En el caso de los docentes, las opiniones están más repartidas, entre los que creen que es poca la revisión que se ha de llevar a cabo y quienes consideran que debe ser mayor (en ambos casos, 46,15\%).

Cuadro 10. Opinión de los diferentes grupos de expertos sobre la necesidad de redefinir las competencias profesionales

\begin{tabular}{|c|c|c|c|c|c|c|c|c|}
\hline \multirow[t]{2}{*}{ Grupo de expertos } & \multirow[t]{2}{*}{ Media } & \multirow{2}{*}{$\begin{array}{l}\text { Desviación } \\
\text { típica }\end{array}$} & \multirow[t]{2}{*}{$\mathrm{CV}^{*}$} & $1^{* *}$ & $2^{* *}$ & $3^{* *}$ & $4^{* *}$ & $\begin{array}{c}\text { No } \\
\text { contesta }\end{array}$ \\
\hline & & & & \multicolumn{5}{|c|}{$(\%)$} \\
\hline Valores globales & 2,85 & 0,699 & 0,245 & 1,79 & 26,79 & 53,57 & 16,07 & 1,79 \\
\hline Profesores & 2,50 & 0,500 & 0,200 & 0 & 46,15 & 46,15 & 0 & 1,79 \\
\hline Profesionales de banca & 3,22 & 0,657 & 0,204 & 0 & 13,04 & 52,17 & 34,78 & 0 \\
\hline Regulares y supervisores & 2,65 & 0,654 & 0,247 & 5 & 30 & 60 & 5 & 0 \\
\hline $\begin{array}{l}{ }^{*} \text { CV } \text { (coeficiente de variació } \\
{ }^{* *} 1=\text { Nada; } 2=\text { Poco; } 3=\text { Ba }\end{array}$ & $4=\mathrm{Muc}$ & & & & & & & \\
\hline
\end{tabular}

Fuente: elaboración propia.

El análisis de las competencias específicas que se han de alcanzar en la formación de los productos derivados financieros de esta investigación se muestra en el cuadro 11, ordenadas según los valores medios obtenidos. 
Cuadro 11. Competencias específicas para el conjunto de expertos

\begin{tabular}{|c|c|c|c|c|c|c|c|}
\hline \multirow{2}{*}{ Competencias } & \multirow{2}{*}{ Media } & \multirow{2}{*}{$\begin{array}{l}\text { Desviación } \\
\text { típica }\end{array}$} & \multirow{2}{*}{$C V^{*}$} & $1 * *$ & $2^{* *}$ & $3^{* *}$ & $4^{* *}$ \\
\hline & & & & \multicolumn{4}{|c|}{$(\%)$} \\
\hline $\begin{array}{l}\text { Capacidad para identificar los tipos de } \\
\text { riesgos financieros }\end{array}$ & 3,84 & 0,367 & 0,096 & 0 & 0 & 16,07 & 83,93 \\
\hline $\begin{array}{l}\text { Comprensión de funcionamiento de los } \\
\text { mercados financieros }\end{array}$ & 3,61 & 0,524 & 0,145 & 0 & 1,79 & 35,71 & 62,50 \\
\hline $\begin{array}{l}\text { Conocimiento de técnicas de gestión y } \\
\text { valoración de instrumentos financieros }\end{array}$ & 3,62 & 0,630 & 0,190 & 1,79 & 3,57 & 55,36 & 39,29 \\
\hline Emitir informes de asesoramiento & 2,70 & 0,777 & 0,288 & 3,57 & 39,29 & 41,07 & 16,07 \\
\hline Modelizar los mercados financieros & 2,49 & 0,735 & 0,295 & 5,36 & 48,21 & 35,71 & 8,93 \\
\hline \multicolumn{8}{|l|}{ * CV (coeficiente de variación). } \\
\hline ** 1 = Nada importante; 2 = Poco import & ite; $3=\ln$ & bortante; $4=1$ & dy impor & & & & \\
\hline
\end{tabular}

Fuente: elaboración propia.

El grupo de expertos considera fundamentales tres de las competencias específicas analizadas, que son, en orden de importancia, las siguientes:

- Capacidad para identificar los tipos de riesgos financieros.

- Comprensión del funcionamiento de los mercados financieros.

- Conocimiento de técnicas de gestión y valoración de instrumentos financieros.

En lo referente a las competencias transversales, en primer lugar, hay que resaltar que se mantiene la elevada valoración que el conjunto de expertos asigna a las competencias analizadas. De esta manera, de un total de 22 competencias, solo 2 se mantienen por debajo de una media de 3 puntos (sobre una escala Likert de 4). La valoración media que el panel de expertos asigna a las competencias transversales se sitúa en 3,37 puntos frente a los 3,19 de las competencias específicas. Por tanto, el panel de expertos considera validadas estas competencias transversales como competencias profesionales de cara al diseño curricular en esta materia. En segundo lugar, haciendo un análisis comparativo entre las diferentes categorías (cognitivas, metodológicas, sociales y personales), en el cuadro 12 se observa que las competencias cognitivas se mantienen, por término medio, como las mejores valoradas (valoración media de 3,52). Las competencias metodológicas alcanzan una valoración media de 3,46 puntos y, en último lugar, se sitúan las competencias de tipo personal $(3,10)$ y las de tipo social $(3,06)$. 
Cuadro 12. Competencias transversales para el conjunto de expertos

\begin{tabular}{l|c|c|c|c|c|c|} 
Competencias & Media & $\begin{array}{c}\text { Desviación } \\
\text { típica }\end{array}$ & $1^{*}$ & $2^{*}$ & $3^{*}$ & $4^{*}$ \\
\cline { 5 - 6 } & & &
\end{tabular}

\section{Competencias de tipo cognitivo (saber)}

\begin{tabular}{|c|c|c|c|c|c|c|}
\hline Capacidad de análisis y síntesis & 3,84 & 0,367 & 0 & 0 & 16,07 & 83,93 \\
\hline Capacidad de aplicar los conocimientos en la práctica & 3,82 & 0,383 & 0 & 0 & 17,86 & 82,14 \\
\hline $\begin{array}{l}\text { Capacidad para la resolución de problemas en entor- } \\
\text { nos nuevos o poco conocidos }\end{array}$ & 3,50 & 0,500 & 0 & 0 & 50 & 50 \\
\hline Capacidad crítica y autocrítica & 3,29 & 0,589 & 0 & 7,14 & 57,14 & 35,71 \\
\hline $\begin{array}{l}\text { Capacidad de pensamiento creativo/Aprendizaje autó- } \\
\text { nomo }\end{array}$ & 3,16 & 0,591 & 0 & 10,71 & 62,50 & 26,79 \\
\hline
\end{tabular}

Competencias de tipo metodológico (saber hacer)

\begin{tabular}{lccccccc}
\hline Habilidad en la búsqueda de información relevante & 3,56 & 0,499 & 0 & 0 & 46,43 & 53,57 \\
\hline $\begin{array}{l}\text { Conocimientos en el uso de TIC } \\
\text { Capacidad de planificación y organización }\end{array}$ & 3,50 & 0,500 & 0 & 0 & 50 & 50 \\
\hline $\begin{array}{l}\text { Habilidad para la gestión y organización de la infor- } \\
\text { mación }\end{array}$ & 3,46 & 0,566 & 0 & 3,57 & 46,43 & 50 \\
\hline \begin{tabular}{l} 
Manejo de programas informáticos especializados \\
\hline
\end{tabular} & 3,16 & 0,576 & 0 & 5,36 & 55,36 & 39,29 \\
\hline
\end{tabular}

Competencias de tipo social (saber estar)

\begin{tabular}{|lcccccc|c|}
\hline Comunicación oral y escrita en la lengua nativa & 3,59 & 0,676 & 1,79 & 5,36 & 25 & 67,86 \\
\hline $\begin{array}{l}\text { Comunicación oral y escrita en la lengua extranjera } \\
\text { Comunicación y expresión matemática, científica y }\end{array}$ & 3,54 & 0,533 & 0 & 1,79 & 42,86 & 55,36 \\
\hline $\begin{array}{l}\text { tecnológica } \\
\text { Liderazgo }\end{array}$ & 2,18 & 0,658 & 1,79 & 8,93 & 58,93 & 30,36 \\
\hline
\end{tabular}




\begin{tabular}{|c|c|c|c|c|c|c|}
\hline \multirow{2}{*}{ Competencias } & \multirow{2}{*}{ Media } & \multirow{2}{*}{$\begin{array}{l}\text { Desviación } \\
\text { típica }\end{array}$} & $1^{*}$ & $2^{*}$ & $3^{*}$ & $4^{*}$ \\
\hline & & & \multicolumn{4}{|c|}{$(\%)$} \\
\hline \multicolumn{7}{|l|}{4} \\
\hline \multicolumn{7}{|l|}{ Competencias de tipo personal (saber ser) } \\
\hline Compromiso ético en el trabajo & 3,50 & 0,598 & 0 & 5,36 & 39,29 & 55,36 \\
\hline Capacidad de tomar decisiones & 3,48 & 0,534 & 0 & 1,79 & 48,21 & 50 \\
\hline Capacidad para trabajar en equipo & 3,48 & 0,627 & 0 & 7,14 & 37,50 & 55,36 \\
\hline Trabajar en entornos de presión & 3,41 & 0,620 & 1,79 & 1,79 & 50 & 46,43 \\
\hline Preocupación por la calidad & 3,38 & 0,614 & 0 & 7,14 & 48,21 & 44,64 \\
\hline Iniciativa y motivación & 3,36 & 0,639 & 0 & 8,93 & 46,43 & 44,64 \\
\hline Habilidad para coordinar grupos de trabajo & 3,07 & 0,530 & 0 & 10,71 & 71,43 & 17,86 \\
\hline Habilidad para la mediación y resolución de conflictos & 2,80 & 0,718 & 1,79 & 32,14 & 50 & 16,07 \\
\hline
\end{tabular}

\subsection{Aspectos relacionados con el proceso de enseñanza-apren- dizaje}

En cuanto a la necesidad de revisar los sistemas de enseñanza, el 44,64\% considera que es necesario llevar a cabo una modificación considerable de los métodos tradicionales de enseñanza; el 39,29 \% opina que es necesario un pequeño cambio; solo el 10,71\% cree que el cambio debe ser profundo; y, finalmente, una minoría considera que hay que dejarlos como están (3,57\%). El grupo de profesionales de banca es el que mayoritariamente ve necesario llevar a cabo esta revisión. Por el contrario, tanto los docentes como los reguladores son partidarios de cambiar poco los métodos de enseñanza.

Atendiendo a la importancia que se le debe otorgar a cada uno de los componentes de las competencias en aras de conseguir una formación de calidad en la materia Derivados Financieros, los expertos confirman la importancia de los conocimientos (46,24\%), bajando levemente el papel de la metodología $(30,45 \%)$ y reafirmando, en último lugar, las cuestiones referidas a actitudes y valores, que se quedan en el $23,31 \%$. 


\subsubsection{Adecuación de los métodos de enseñanza}

Se confirman los contenidos teóricos con «clases magistrales» (23,78\%), seguidas de las «clases prácticas» $(22,67 \%)$, como los métodos idóneos para la enseñanza de esta materia, aumentando ligeramente la valoración media con respecto a la primera ronda.

Con menores puntuaciones quedan el resto de métodos: «prácticas externas» en empresas $(14,93 \%)$ y «estudio individual» $(13,29 \%)$. Las «tutorías» $(7,27 \%)$ y el «trabajo en grupo" $(7,46 \%)$ son las técnicas a las que, por el contrario, se les da menos importancia (véase cuadro 13).

Cuadro 13. Opinión del conjunto de expertos sobre el reparto porcentual de los métodos de enseñanza

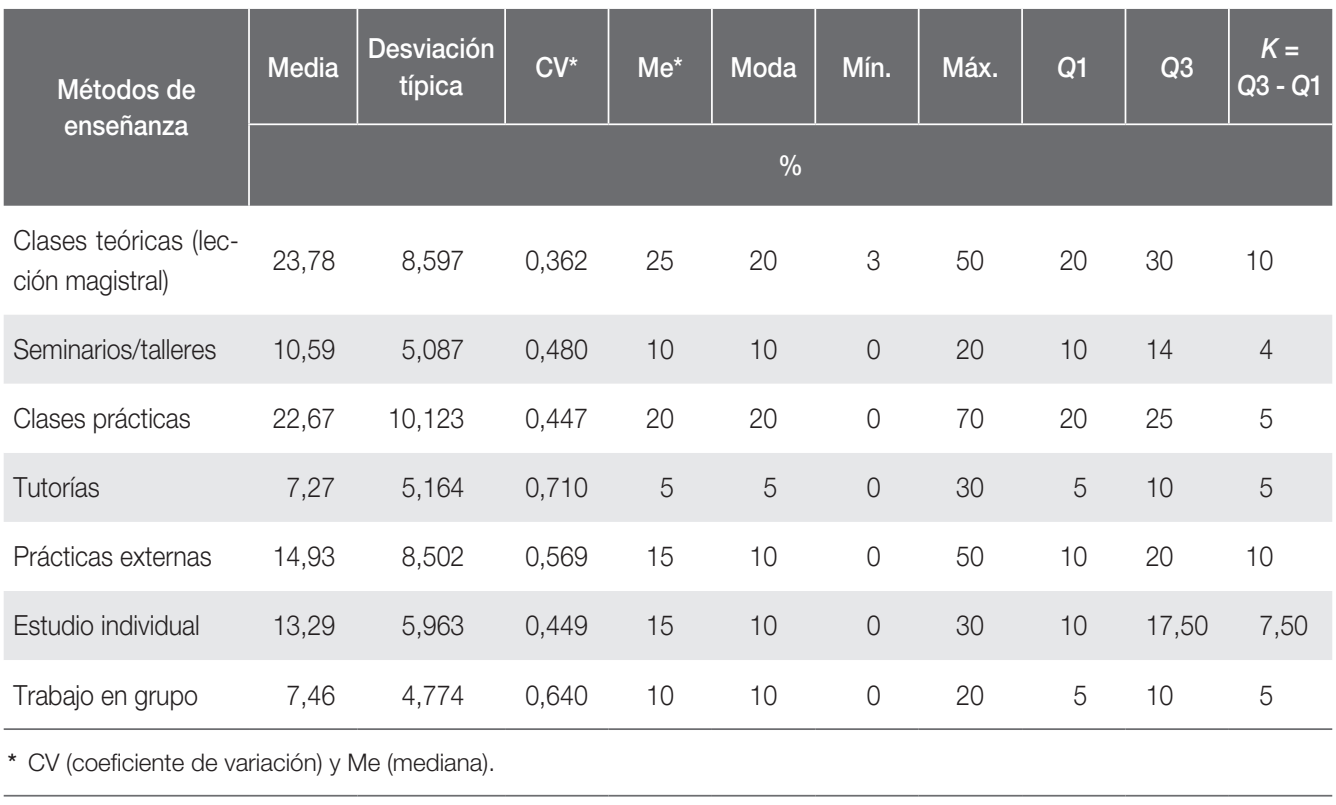

Analizando los resultados anteriores según los grupos de expertos participantes, se aprecian algunas diferencias. Así, en el grupo de profesores, las «clases teóricas» alcanzan el nivel máximo $(27,31 \%)$ y el más bajo en esta modalidad se obtiene en el grupo de profesionales de banca $(20,77 \%)$.

En cuanto a las "clases prácticas", son los profesionales de banca los que las valoran mejor $(25,23 \%)$, seguidos de los docentes $(24,62 \%)$ y de los reguladores $(18,60 \%)$. 
Para el colectivo de profesores, el «trabajo en grupo» (5,69\%) resulta el método de enseñanza menos adecuado, seguido de las «tutorías» (8,46\%). Asimismo, son los que peor valoración otorgan a las «prácticas externas».

\subsubsection{Sistema de evaluación}

Los expertos se decantan por la necesidad de introducir cambios en los sistemas de evaluación que se tienen que emplear en la enseñanza de los derivados financieros. De esta manera, la mitad del panel (50\%) considera que los sistemas de evaluación deben cambiar «poco», mientras que un $32,14 \%$ opina que deben cambiar «bastante». Tan solo el $7,14 \%$ de los expertos piensa que no deben cambiar «nada» y el 8,93\% que deben cambiar «mucho».

Como se muestra en el cuadro 14 en una escala Likert de cuatro opciones ( 1 = Nada eficaz; 2 = Poco eficaz; 3 = Eficaz; 4 = Muy eficaz), solo tres de los sistemas de evaluación analizados superan los 3 puntos. La valoración más alta se mantiene en las «Pruebas de respuesta corta» $(3,33)$ y le siguen los «Trabajos y proyectos individuales» $(3,15)$, que se sitúan ligeramente por delante de las «Pruebas objetivas (tipo test)» $(3,11)$. Por el contrario, las peores valoraciones las mantienen los «Sistemas de autoevaluación» $(2,31)$ y las «Pruebas orales» $(2,27)$.

Cuadro 14. Eficacia de los sistemas de evaluación

\begin{tabular}{|c|c|c|c|c|c|c|c|}
\hline \multirow{2}{*}{ Sistema de evaluación } & \multirow{2}{*}{ Media } & \multirow{2}{*}{$\begin{array}{l}\text { Desviación } \\
\text { típica }\end{array}$} & \multirow{2}{*}{$C V^{*}$} & $1^{* *}$ & $2^{* *}$ & $3^{* *}$ & $4^{* *}$ \\
\hline & & & & \multicolumn{4}{|c|}{$(\%)$} \\
\hline Pruebas de respuesta corta & 3,33 & 0,605 & 0,182 & 0 & 7,27 & 52,73 & 40 \\
\hline Trabajos y proyectos individuales & 3,15 & 0,724 & 0,230 & 3,64 & 9,09 & 56,36 & 30,91 \\
\hline Pruebas objetivas (tipo test) & 3,11 & 0,593 & 0,191 & 0 & 12,73 & 63,64 & 23,64 \\
\hline Pruebas con simuladores & 2,95 & 0,749 & 0,254 & 0 & 30,91 & 43,64 & 25,45 \\
\hline Pruebas de respuesta larga & 2,75 & 0,768 & 0,280 & 1,82 & 40 & 40 & 18,18 \\
\hline Trabajos y proyectos en grupo & 2,67 & 0,854 & 0,320 & 7,27 & 36,36 & 39,18 & 18,18 \\
\hline $\begin{array}{l}\text { Realización de informes/memorias de } \\
\text { prácticas }\end{array}$ & 2,56 & 0,707 & 0,276 & 3,64 & 45,45 & 41,82 & 9,09 \\
\hline
\end{tabular}




\begin{tabular}{|c|c|c|c|c|c|c|c|}
\hline \multirow{2}{*}{ Sistema de evaluación } & \multirow{2}{*}{ Media } & \multirow{2}{*}{$\begin{array}{l}\text { Desviación } \\
\text { típica }\end{array}$} & \multirow{2}{*}{$\mathrm{CV}^{*}$} & $1^{* *}$ & $2^{* *}$ & $3^{* *}$ & $4^{* *}$ \\
\hline & & & & \multicolumn{4}{|c|}{ (\%) } \\
\hline \multicolumn{8}{|l|}{4} \\
\hline Sistemas de autoevaluación & 2,31 & 0,806 & 0,349 & 12,73 & 52,73 & 25,45 & 9,09 \\
\hline Pruebas orales & 2,27 & 0,645 & 0,284 & 5,45 & 67,27 & 21,82 & 5,45 \\
\hline
\end{tabular}

Fuente: elaboración propia.

\section{Discusión y conclusiones}

Como se ha puesto de manifiesto en la revisión bibliográfica realizada, para el diseño del plan de estudios en la materia Derivados Financieros se debe contar con la opinión de los expertos. A este respecto, la validez de los resultados de esta investigación está garantizada por los años de ejercicio profesional y la experiencia docente de los participantes.

Los resultados de este estudio confirman los obtenidos por De Miguel et al. (2005) en cuanto a la distribución general de conocimientos, procedimientos y actitudes a la hora de analizar los componentes de las competencias académicas. De esta forma, se otorga más importancia a los conocimientos en detrimento de los aspectos metodológicos. Por el contrario, los resultados difieren en cuanto a la importancia de los sistemas de evaluación.

En relación a la definición de los contenidos que se han de estudiar, así como el número de créditos ECTS que se le debe asignar a esta materia, los resultados están en función del grado estudiado. La respuesta del panel muestra concordancia con la valoración otorgada en los puntos anteriores (inclusión en el plan de estudios y obligatoriedad de la asignatura). En cuanto a la distribución entre contenidos teóricos y prácticos para el estudio de esta asignatura aconsejan una distribución equilibrada, con leve predominio de la formación práctica.

La respuesta mayoritaria del panel de expertos manifiesta estar de acuerdo al considerar conveniente el diseño del plan de estudios en torno a la adquisición de competencias profesionales como factor determinante a la hora de facilitar la empleabilidad y el desarrollo personal y profesional de los egresados. Al mismo tiempo, aconsejan una redefinición importante de las competencias debido a los cambios producidos en materia de regulación financiera en los últimos años. Son los profesionales de banca los más firmes partidarios de llevar a cabo esta redefinición. Por el contrario, el grupo de docentes no ve necesario introducir importantes modificaciones en las competencias existentes. 
En lo referente a las competencias transversales, se debe prestar más atención a las competencias cognitivas, seguidas de las metodológicas, las de tipo personal y, en último lugar, las de tipo social. Incluso se les llega a otorgar más importancia que a algunas de las competencias específicas, por lo que deben ser consideradas con mayor prioridad a la hora de llevar a cabo el diseño curricular en esta materia.

En lo relativo al peso porcentual que debe asignarse a cada uno de los componentes de las competencias para alcanzar una formación adecuada en la materia Derivados Financieros, los expertos se inclinan por dar más importancia a los conocimientos sobre la materia, algo menos a la metodología y en menor medida a las actitudes y valores.

Por último, los cambios introducidos en la regulación financiera que ha tenido lugar en los últimos años hacen necesaria una revisión de los métodos de enseñanza, tanto de los sistemas de enseñanza como de los sistemas de evaluación tradicionales.

De forma mayoritaria los expertos se decantan por las clases magistrales y por las clases prácticas como los métodos de enseñanza idóneos para esta materia, complementando con las prácticas externas en empresas y el estudio individual. Por el contrario, las tutorías y el trabajo en grupo son los métodos a los que se les da menos importancia.

Los sistemas actuales de evaluación que los expertos destacan como eficaces en la valoración de los rendimientos del alumnado son las pruebas de respuesta corta, las pruebas objetivas (test) y los trabajos individuales. Por el contrario, las peores valoraciones las reciben los sistemas de «autoevaluación» y las «pruebas orales».

De acuerdo con lo expuesto anteriormente, y con el objetivo de conseguir una formación de calidad en la materia Derivados Financieros acorde a los requerimientos del mercado laboral y a las exigencias de los profesionales que hacen uso de estos productos, se realizan las siguientes «propuestas de mejora»:

- Consideración de la materia Derivados Financieros como asignatura obligatoria en todos los grados.

- Revisión de los contenidos que deben incluirse en el temario de la asignatura, incluyendo los instrumentos más importantes que se emplean en este mercado.

- Recomendar el estudio de la asignatura con carácter específico, más allá de unos conceptos generales básicos que solo pretendan explicar su funcionamiento, al mismo tiempo que prestar más atención a los riesgos asociados a este tipo de instrumentos.

- Redefinir las competencias, adecuándolas a las exigencias de los expertos y otorgando más importancia a las competencias específicas relacionadas con la identificación de riesgos y funcionamiento de los mercados. En cuanto a las 
competencias transversales, se debe hacer más énfasis en las de tipo cognitivo y metodológicas y, en menor medida, en las personales y sociales. También es necesario ajustar la distribución entre contenidos teóricos y prácticos, asignando una mayor importancia a los aspectos prácticos de la asignatura.

- Considerar las clases magistrales como el sistema de enseñanza más importante, junto a la realización de casos prácticos. Rebajar la ponderación del examen para situarlo en unos niveles del $60 \%$ de la nota final. En cuanto al tipo de preguntas que hay que realizar, los expertos aconsejan las pruebas de respuesta corta y las pruebas objetivas (tipo test).

- Es necesario que los equipos docentes trabajen en la redefinición de los planes formativos de todas las asignaturas que permitan una formación integral en la materia Derivados Financieros en las titulaciones de todos los grados del área de economía y empresa.

Por último, conviene resaltar algunas «limitaciones» en la investigación realizada:

- En primer lugar, hay que destacar la ausencia de estudios e investigaciones realizadas hasta ahora en este sentido en el área referida a los instrumentos financieros derivados.

- También conviene indicar que, si bien el grupo de profesionales de banca y reguladores se puede considerar ampliamente representado, solo se ha contado con académicos de las universidades madrileñas.

Siendo conscientes de las limitaciones antes mencionadas, la presente investigación pretende considerarse como un paso más en el reto de la universidad en la constante reforma curricular de los grados universitarios, en aras de conseguir una formación de calidad ajustada a las necesidades de una sociedad en continuo cambio, con unos egresados universitarios profesionales con unas competencias adquiridas que permitan su adaptación a la nueva realidad del mercado en continua evolución, evidenciada, en parte, por la crisis financiera reciente.

Esta reforma en los programas educativos pretende contribuir a la creación de profesionales más competitivos, integrados y eficientes en el seno de la Unión Europea.

Teniendo en cuenta las conclusiones previas se podría realizar una propuesta de diseño curricular para la enseñanza de los derivados financieros, adecuada a las exigencias que el mercado demanda. 


\section{Referencias bibliográficas}

Bailie, J. (2015). Online graduate instruction: what faculty consider reasonable in relation to what students expect. Journal of Online Learning and Teaching, 11(1), 42-54.

Bisquerra Alzina, R. y Pérez-Escoda, N. (2015). ¿Pueden las escalas Likert aumentar en sensibilidad? REIRE. Revista d'Innovació i Recerca en Educació, 8(2), 129-147.

Cabero Almenara, J. e Infante Moro, A. (2014). Empleo del método Delphi y su empleo en la investigación en comunicación y educación. EDUTEC. Revista Electrónica de Tecnología Educativa, 48, 1-16. https://doi.org/10.2155 6/edutec.2014.48.187

Chuenjitwongsa, S., Oliver, R. G. y Bullock, A. D. (2017). Developing educators of European undergraduate dental students: towards an agreed curriculum. European Journal of Dental Education, 22(3), 179-191. https://doi.org/ 10.1111/eje.12306

Cruz Ramírez, M. y Rúa Vásquez, J. A. (2018). Surgimiento y desarrollo del método Delphi: una perspectiva cienciométrica. Biblios, 71 , 90-107.

Dávila Quintana, C. D., Mora, J. G., Pérez Vazquez, P. J. y Vila Lladosa, L. E. (2016). La cooperación universidad-empresa, instrumento para mejorar las competencias de los egresados. Investigaciones de Economía de la Educación, 11(11), 265-282.

Freire-Seoane, M. ${ }^{a}$ J., Teijeiro-Álvarez, M. M. y País Montes, C. (2013). La adecuación entre las competencias adquiridas por los graduados y las requeridas por los empresarios. Revista Educación, 362, 13-41.

Fullana Noell, J., Pallisera Díaz, M. ${ }^{a}$ y Planas Lladó, A. (2011). Las competencias profesionales de los educadores sociales como punto de partida para el diseño curricular de la formación universitaria. Un estudio mediante el método Delphi. Revista Iberoamericana de Educación, 56(1), 1-13.

García Aracil, A. y Palomares Montero, D. (2012). Indicadores para la evaluación de las instituciones universitarias: validación a través del método Delphi. Revista Española de Documentación Científica, 35(1), 119-144.

George, C. y Trujillo, L. (2018). Aplicación del método Delphi modificado para la validación de un cuestionario de incorporación de las TIC en la práctica docente. Revista Iberoamericana de Evaluación Educativa, 11(1), 113-134. https://doi.org/10.15366/riee2018. 11.1 .007

Gutiérrez García, J. J., Pérez Riquelme, F., López Rojo, C. y López Alegría, C. (2003). Necesidades y problemas para el desarrollo de la educación para la salud en la escuela: estudio Delphi en la región de Murcia. Serie Educación para la Salud. Informe 36. Consejería de Sanidad.

Herrero Curiel, E. (2018). Rethinking journalism education in Spain: the gap between university studies and the labour market. European Journal of Social Sciences Education and Research Articles, 5(2), 61-72.

Homberg, A., Klafke, N., Glassen, K., Loukanova, S. y Mahler, C. (2020). Role competencies in interprofessional undergraduate education in complementary and integrative medicine: a delphi study. Complementary Therapies in Medicine, 54. https://doi.org/10. 1016/j.ctim.2020.102542

Hynes, W. y Kwon, H. J. (2018). An Evaluation of oral presentation competency in interior design education. International Journal of Art \& Design Education, 37(3), 387- 398. https:// doi.org/10.1111/jade.12134

Juanas Oliva, A. de, Martín del Pozo, R. y González-Ballesteros, M. (2016). Competencias docentes para desarrollar la competencia 
científica en educación primaria. Bordón. Revista de Pedagogía, 68(3), 103-120.

Landeta, J. (1999). El método Delphi. Ariel.

Lnenicka, M., Hana, K., Renata, M. y Jitka, K. (2020). Big and open linked data analytics: a study on changing roles and skills in the higher educational process. International Journal of Educational Technology in Higher Education, 17(8). https://doi.org/10.1186/s4 1239-020-00208-z

López-Gómez, E. (2018). El método Delphi en la investigación actual en educación: una revisión teórica y metodológica. Educación XX1, 21(1), 17-40.

López Meneses, E. J., Bernal Bravo, C., Leiva Olivencia, J. J. y Martín Padilla, A. H. (2018). Validación del instrumento didáctico de valoración de observatorios digitales sobre MOOC mediante el método Delphi. Campus Virtuales, 7(1), 95-110.

Miguel, M. de (Dir.) Alfaro, I. J., Apodaca, P., Arias, J. M., García, E. y Pérez, A. (2005). Modalidades de enseñanza centradas en el desarrollo de competencias. Orientaciones para promover el cambio metodológico en el EEES. MEC/Universidad de Oviedo.

Pozo Muñoz, C. y Bretones Nieto, B. (2015). Dificultades y retos en la implantación de los títulos de grado en las universidades españolas. Revista de Educación, 367, 181-147. https://doi.org/10.4438/1988-592X-RE-2 015-367-286

Rosales, C. y Cabrera, L. (2017). Causas y soluciones de los bajos resultados educativos de Canarias mostrados en los informes de evaluación. Revista Qurriculum, 30, 157-181.
Steurer, J. (2011). The Delphi method: an efficient procedure to generate knowledge. Skeletal Radiology, 40(8), 959-961. https://doi. org/10.1007/s00256-011-1145-z

Torres-Coronas, T. y Vidal-Blasco, M. ${ }^{a}$ A. (2015). Percepción de estudiantes y empleadores sobre el desarrollo de competencias digitales en la educación superior. Revista de Educación, 367, 63-90.

Uztosun, M. S. (2018). Professional competences to teach english at primary schools in Turkey: a Delphi study. European Journal of Teacher Education, 41(4), 549-565. https:// doi.org/10.1080/02619768.2018.1472569

Vallés, M. (1997). Técnicas cualitativas de investigación social: reflexión metodológica y práctica profesional. Síntesis.

Victoria, M., Franziska, H., Per, B. y Westhuizen. C. van der. (2020). Challenges and contexts in establishing adaptive learning in higher education: findings from a Delphi study. International Journal of Educational Technology in Higher Education, 17(1). https://doi.org/10. 1186/s41239-020-00209-y

Yağci, E. y Güneyli, A. (2018). An analysis of the professional competencies of Turkish language and literature teachers on the basis of the ability of using and managing technology. EURASIA Journal of Mathematics, Science and Technology Education, 14(7), 3.389-3.404

Yániz, C. (2008). Las competencias en currículo universitario: implicaciones para diseñar el aprendizaje y para la formación del profesorado. Revista de Docencia Universitaria, 6(1). https://doi.org/10.4995/redu.2008.6281 